\title{
The Effectiveness of Mindfulness Intervention on Response Inhibition in Children With Attention Deficit/Hyperactivity Disorders
}

\author{
Meysam Rezayi, Bagher sardary*
}

Department of Educational Psychology, Tabriz Branch, Islamic Azad University, Tabriz, Iran Article Info:

\section{A BSTRACT}

Introduction: Failure in response inhibition has been the underlying impairment is attention deficit/ hyperactivity disorder and constitute the core of the disruption of processing processes in the executive functions of this disorder. The purpose of this study was to determine the effectiveness of mindfulness intervention on response inhibition in children with attention deficit/hyperactivity disorders. Materials and Methods: The present s tudy is a semi-experimental study with pretest-posttest and control group design. The statis tical population of the study included all male students studying in the secondary schools of Maku city in the academic year 2018-2019. From this population, a sample of 24 people was selected by purposeful sampling method using Conner's parent rating scale (CPRS-48) and were randomly divided into two experimental and control groups. The GO/NO-GO tests was performed on the participants in two stages pre-test and post-test. Data analysis was performed using covariance analysis. Results: Data analysis showed that mindfulness intervention led to a significant difference between groups in response inhibition of $64 \%$ and its components including omission error 39\%, commision error 44\% and reaction time 46\%. Conclusion: Mindfulness intervention is a suitable strategy to improve response inhibition in children with attention deficit/ hyperactivity disorder and can be used as an effective intervention.

\section{Keywords: \\ 1. Mindfulness \\ 2. Attention \\ 3. Population}

*Corresponding Author: Bagher sardary

E-mail: sardary1152bagher@gmail.com 


\title{
اثر بخشى مداخله ذهن آكاهى بر بازدارى ياسخ در كودكان با علايم نقص توجه /بيشفعالى
}

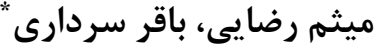

گروه روانشناسى تربيتى، واحد تبريز، دانشخاه آزاد اسلامى، تبريز، ايران

\section{جـ جـ ـ}

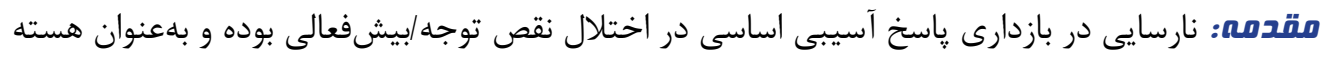

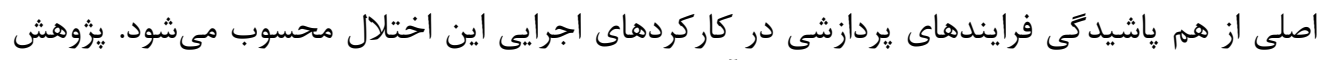

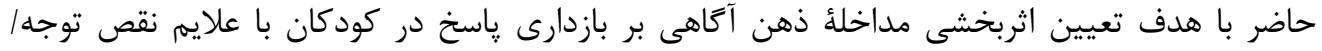

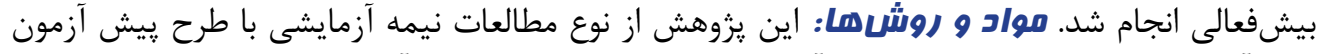

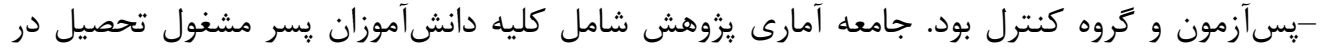

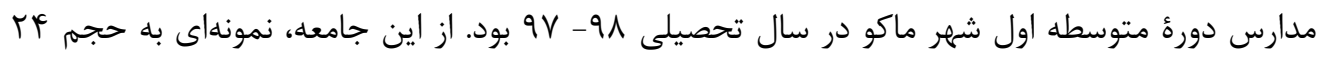

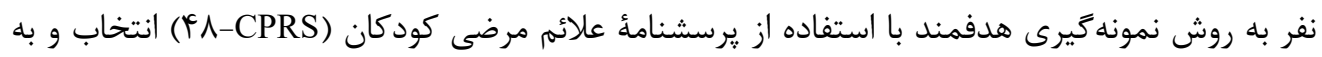

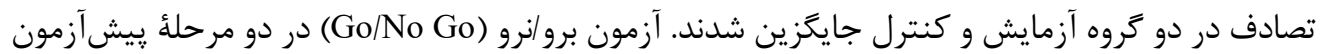

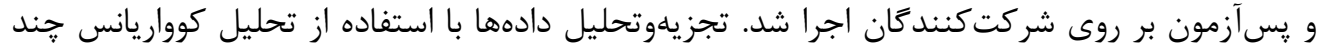

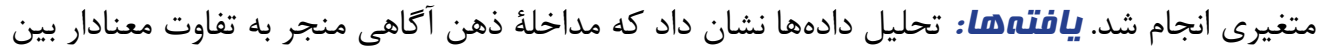

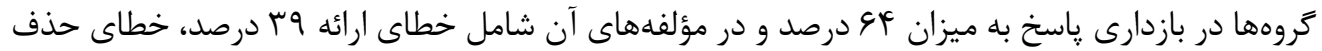

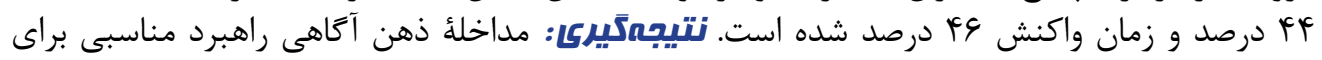

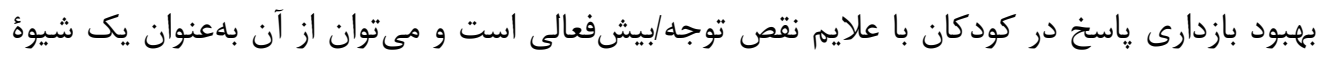
مداخلهاى موثر استفاده نمود.

: إز إزهاى كليدى:

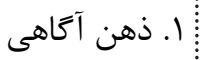
(r) (r) 


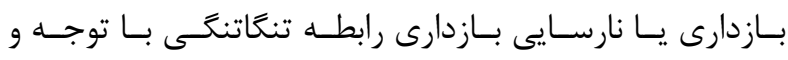
مقدمه

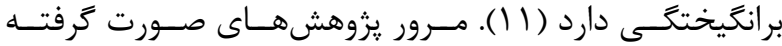

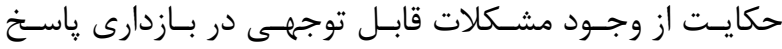

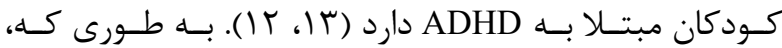

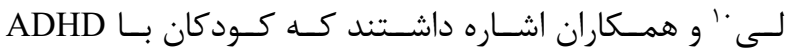

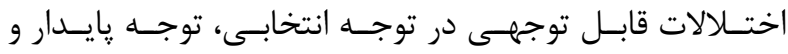

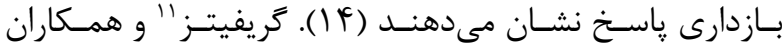

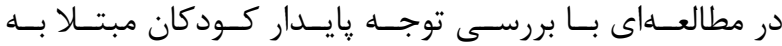

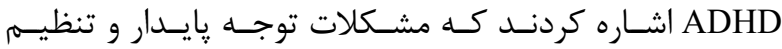

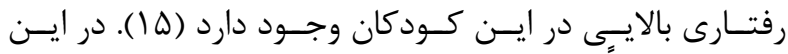

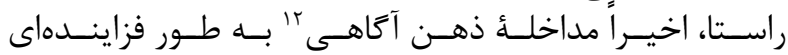

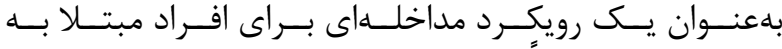

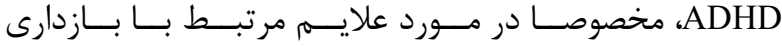

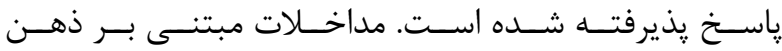

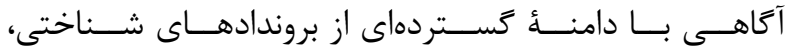

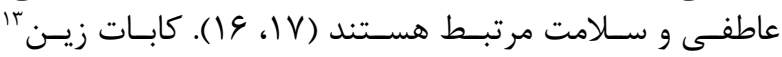

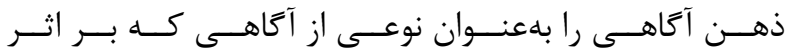

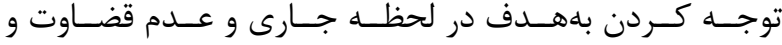

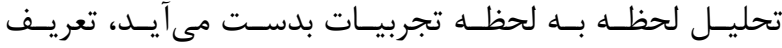

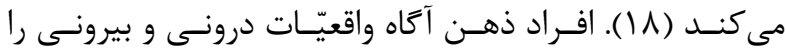

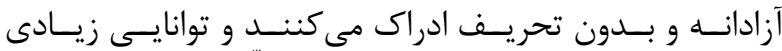

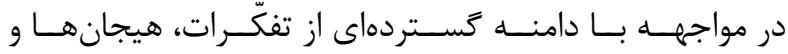

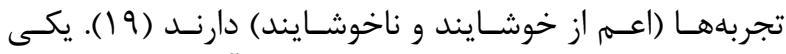

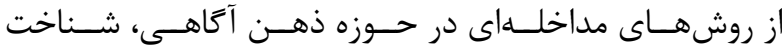

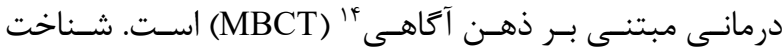

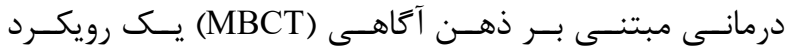

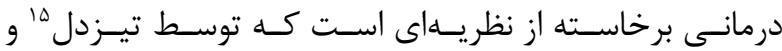

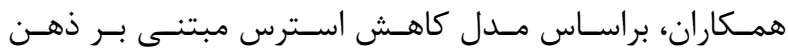

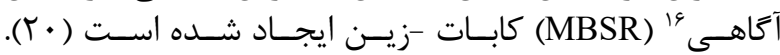

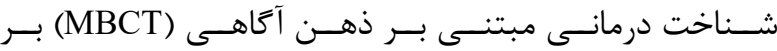

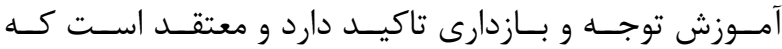

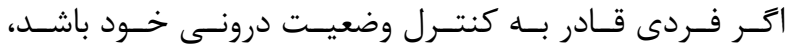

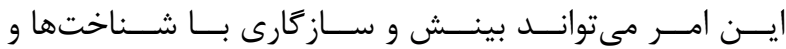

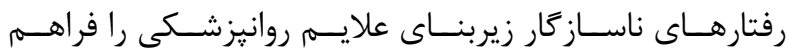

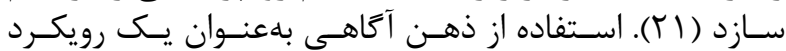

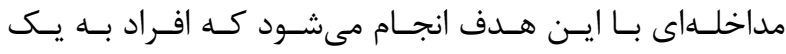

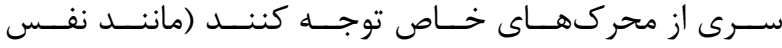

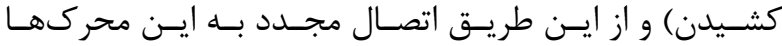

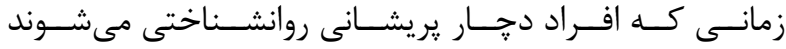

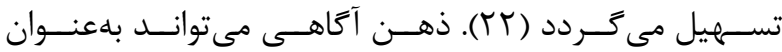

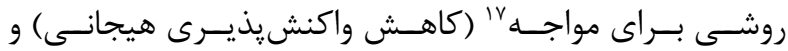

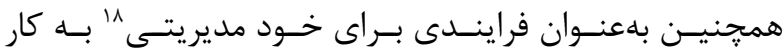

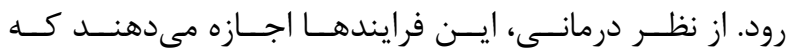

${ }^{1}$ Neurodevelopmenta

${ }^{2}$ Diagnostic and statistical manual of mental disorders

${ }^{3}$ Excutive functions

${ }^{4}$.Prefrontal cortex

${ }^{5}$ Response Inhibition

${ }^{6}$ Prepotent

${ }^{7}$ Ongoing

${ }^{8}$ Interference-control

${ }^{9}$ Wang

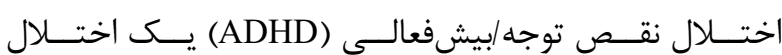

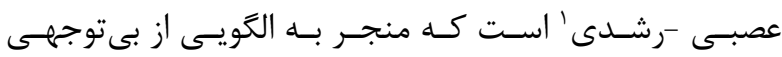

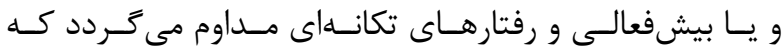

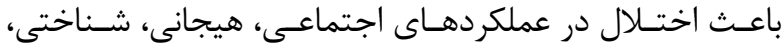

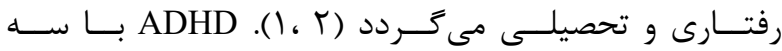

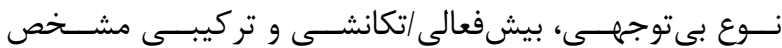

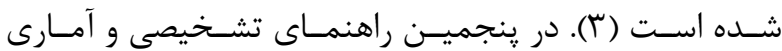

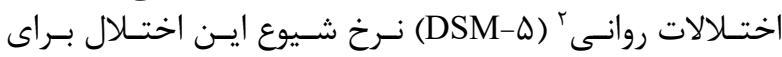

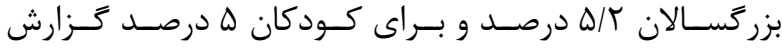

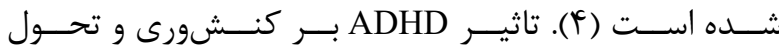

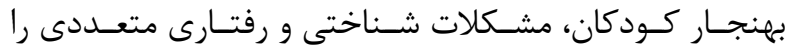

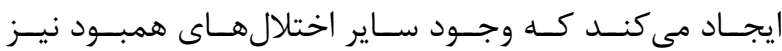

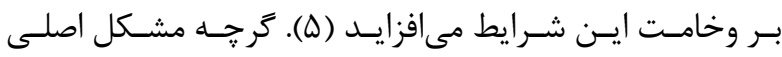

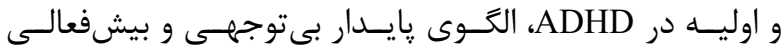

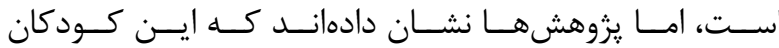

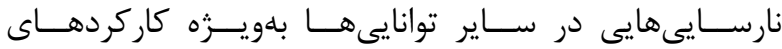

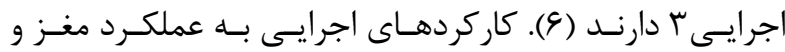

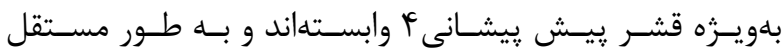

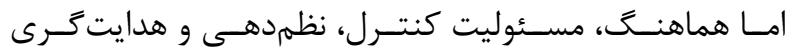

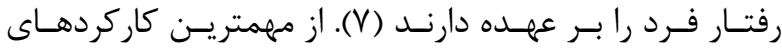

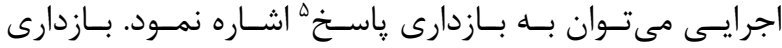

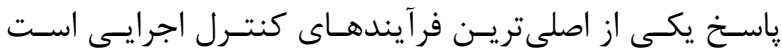

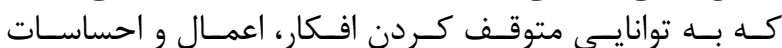

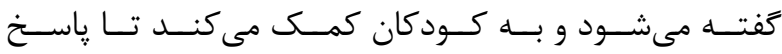

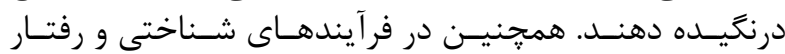

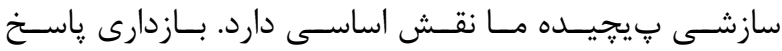

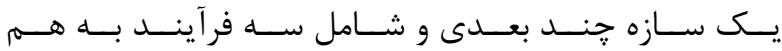

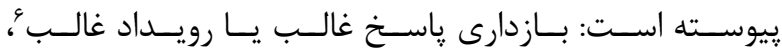

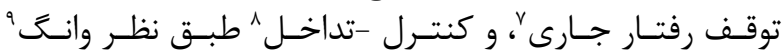

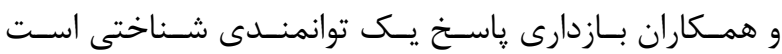

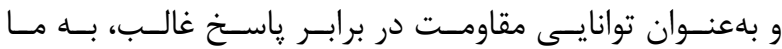

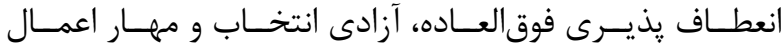

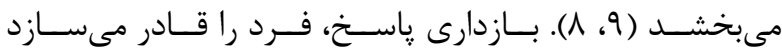

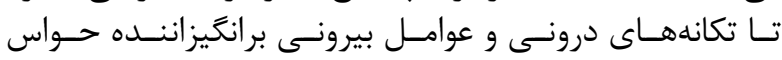

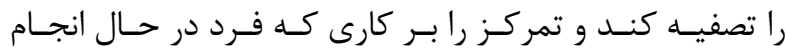

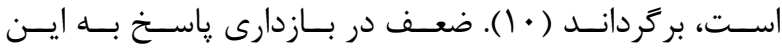

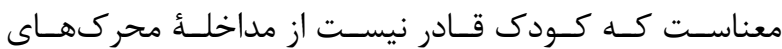

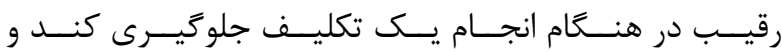

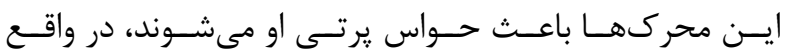

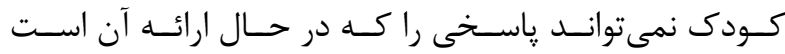

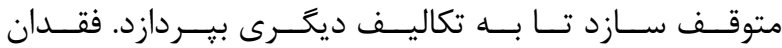

${ }^{10}$ Lee

${ }^{11}$ Griffiths

${ }^{12}$ Mindfulness intervention

${ }^{13}$ Kabat-Zinn

${ }^{14}$ Mindfulness- Based Cognitive Therapy (MBCT)

${ }^{15}$ Teasdale, Segal \& Williams

${ }^{16}$ Mindfullness-Based Stress Reduction (MBSR)

${ }^{17}$ Exposure technique

${ }^{18}$ Process of self-management 


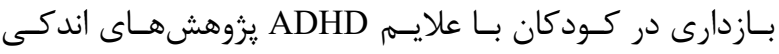

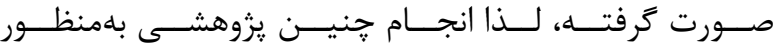

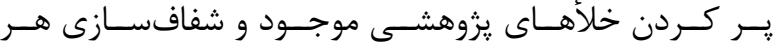

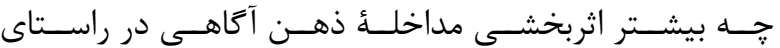

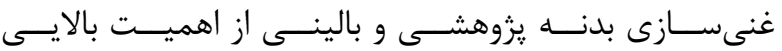

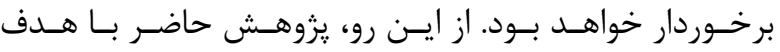

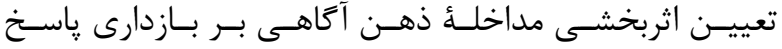

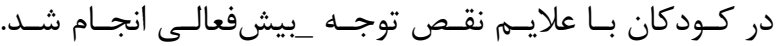

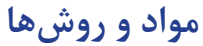

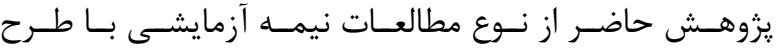

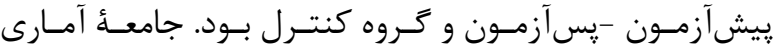

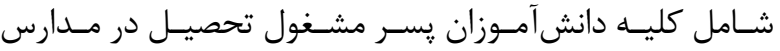

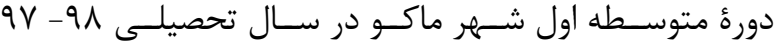

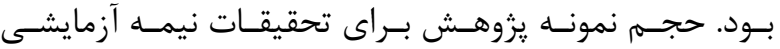

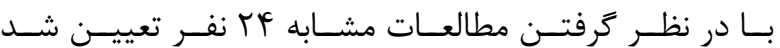

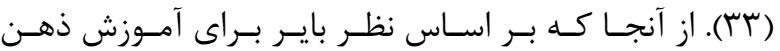

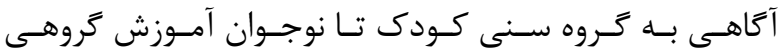

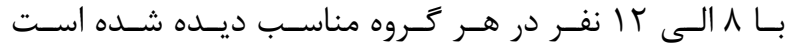

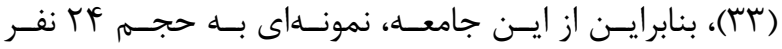

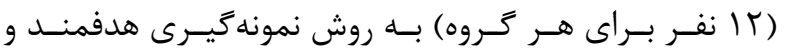

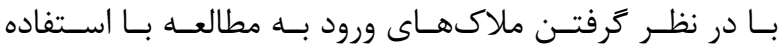

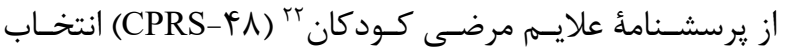

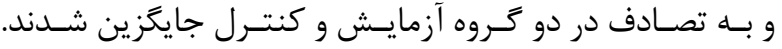

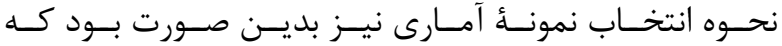

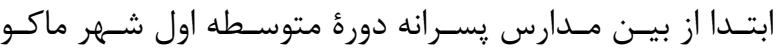

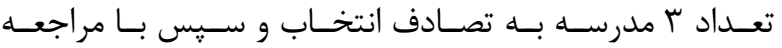

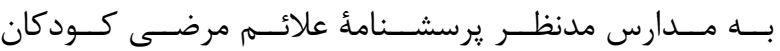

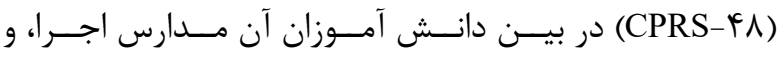

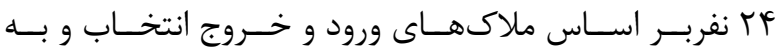

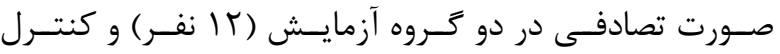

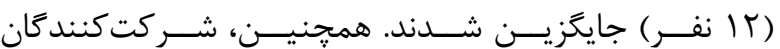

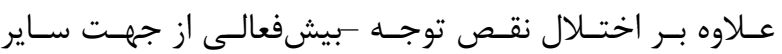

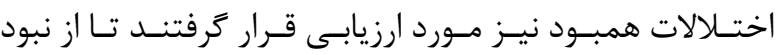

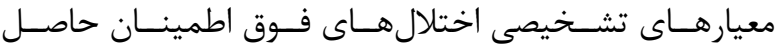

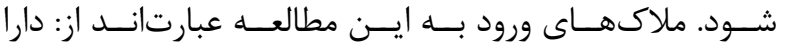

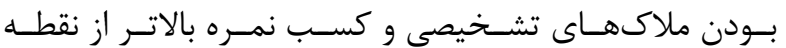

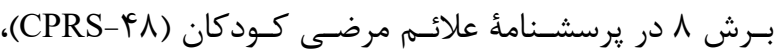

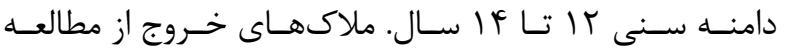

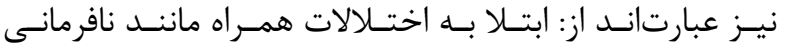

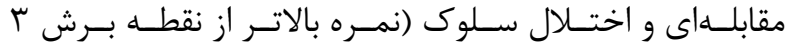

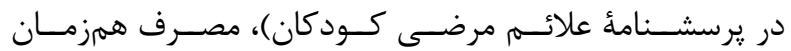

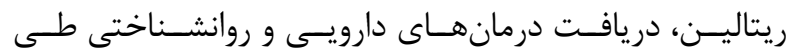

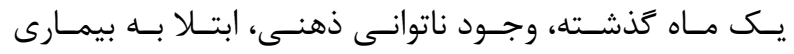

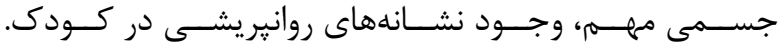

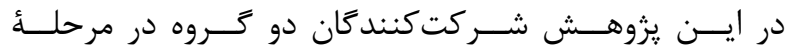

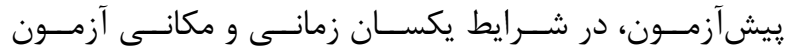

\footnotetext{
${ }^{19}$ Bishop

${ }^{20}$ Self-regulation of attention
}

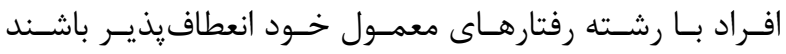

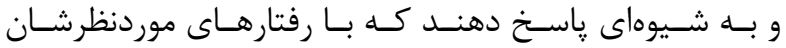

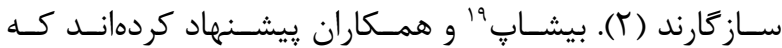

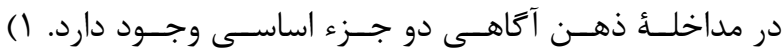

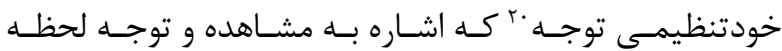

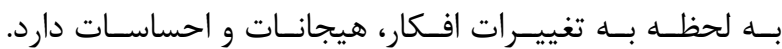

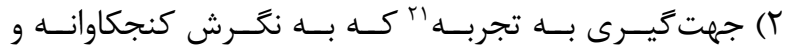

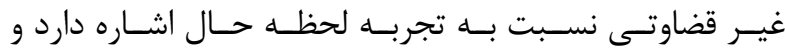

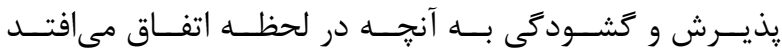

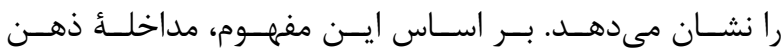

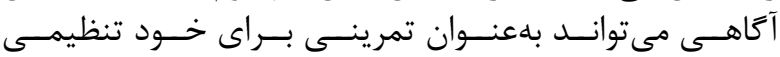

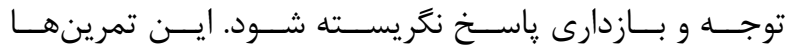

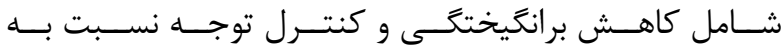

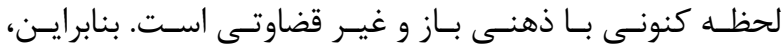

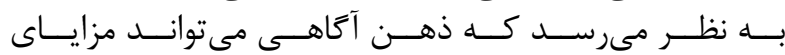

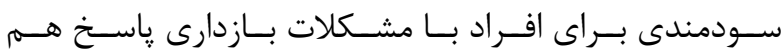

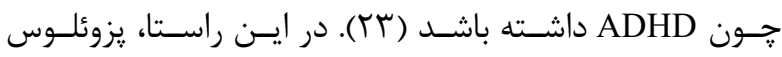

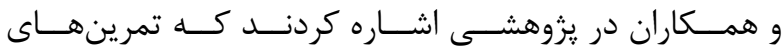

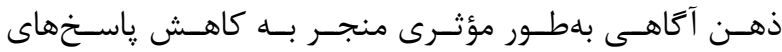

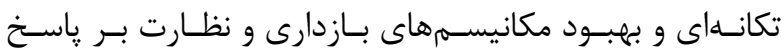

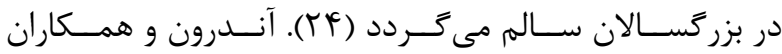

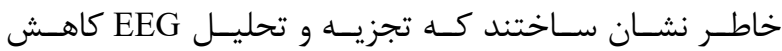

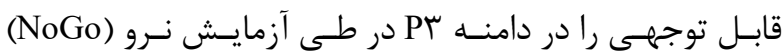

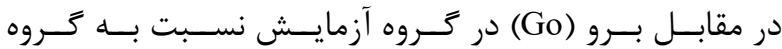

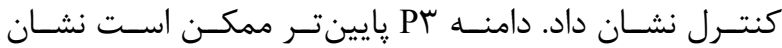

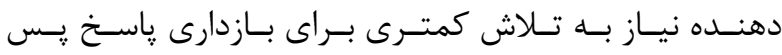

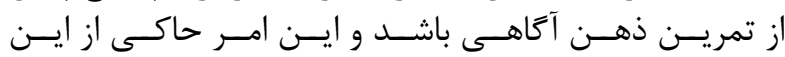

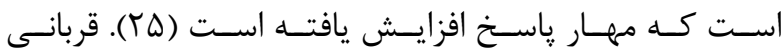

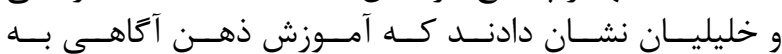

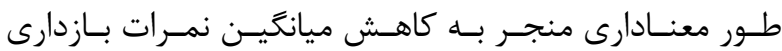

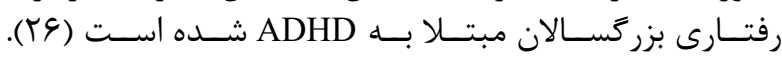

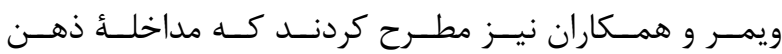

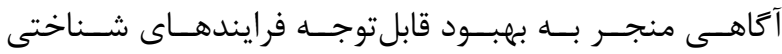

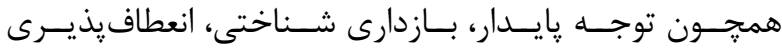

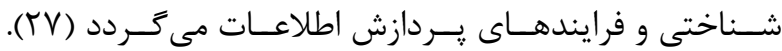

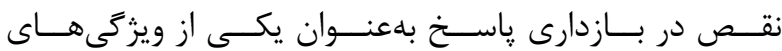

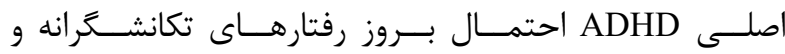

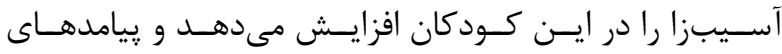

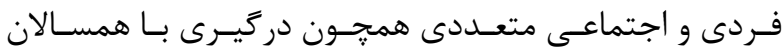

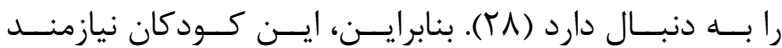

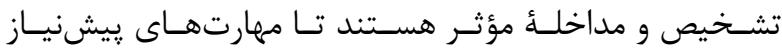

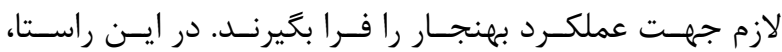

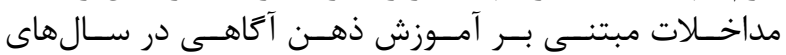

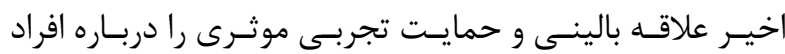

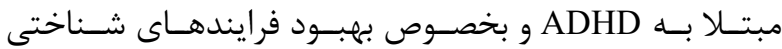

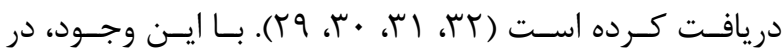

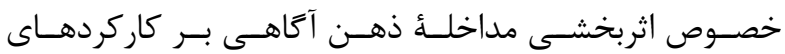

\footnotetext{
${ }^{21}$ Orientation to experience

${ }^{22}$ Conner's parent rating scale (CPRS)
} 


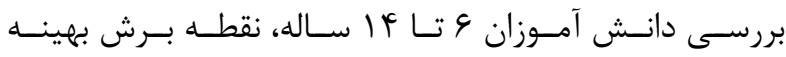

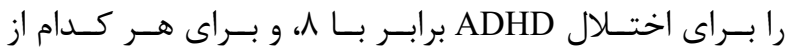

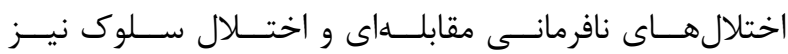

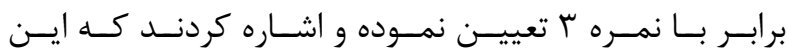

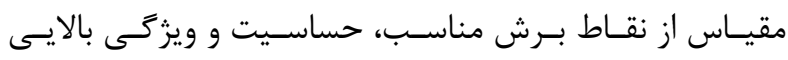

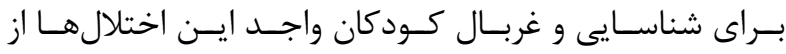

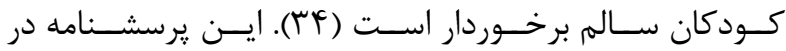

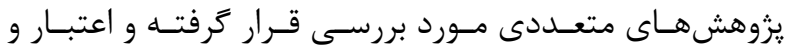

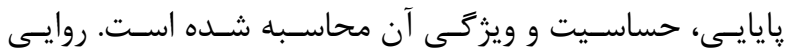

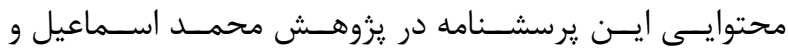

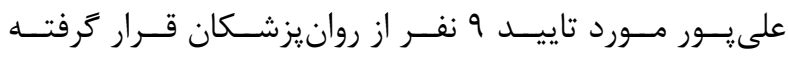

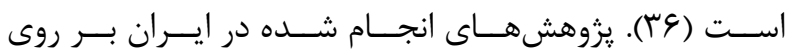

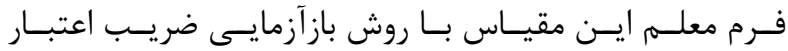

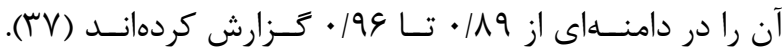

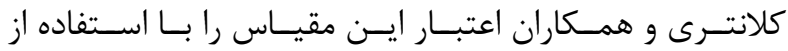

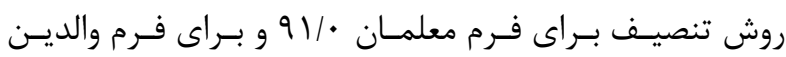

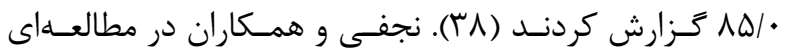

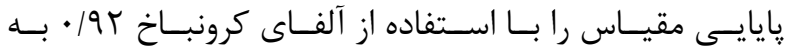

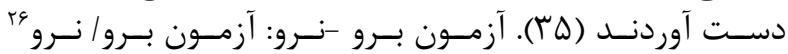

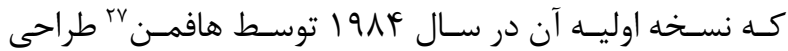

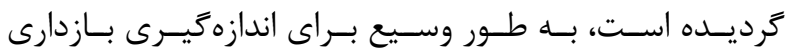

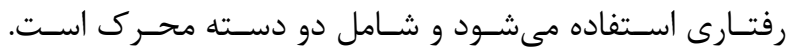

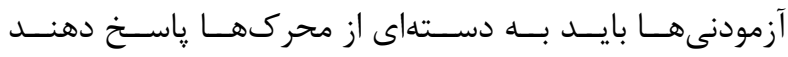

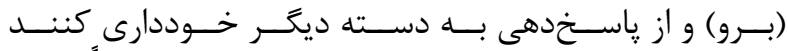

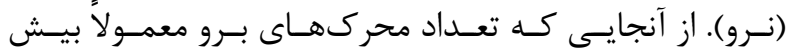

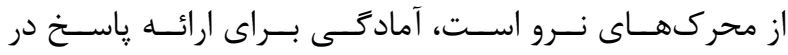

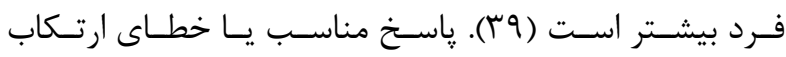

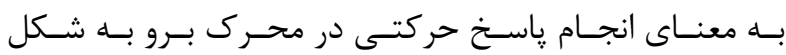

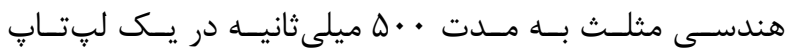

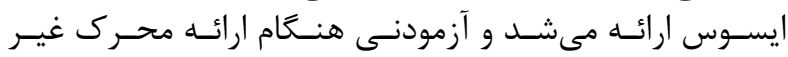

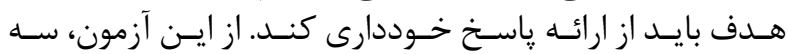

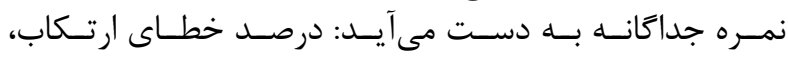

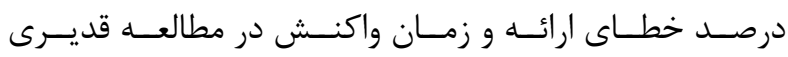

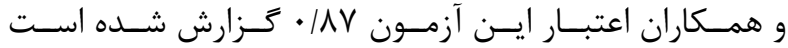

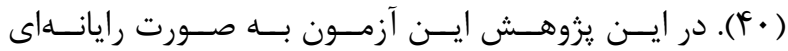

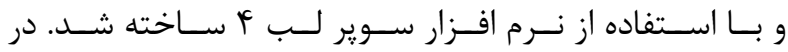

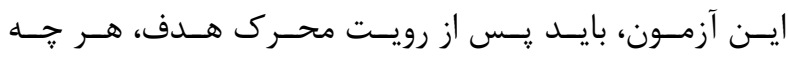

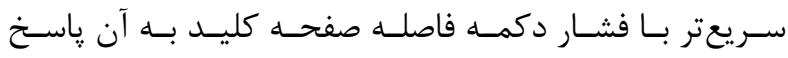

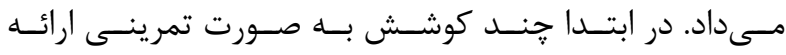

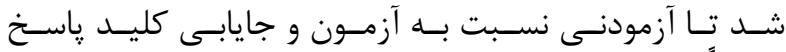

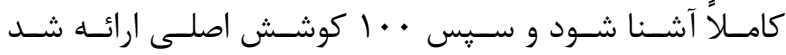

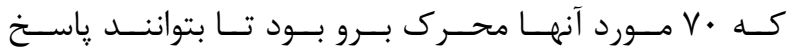

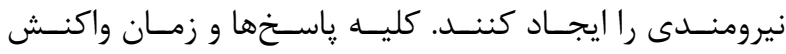

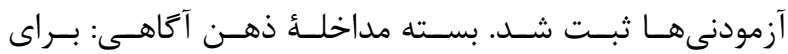

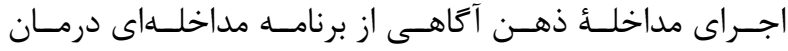

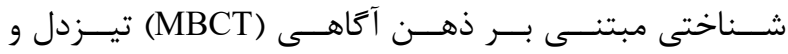

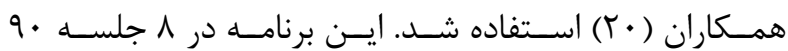

${ }^{23}$ Children symptom inventory

${ }^{24}$ Slug

${ }^{25}$ Oppositional defaint disorder (ADD)

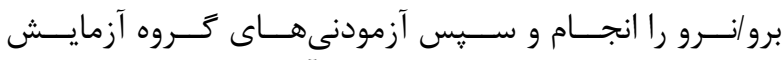

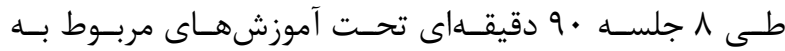

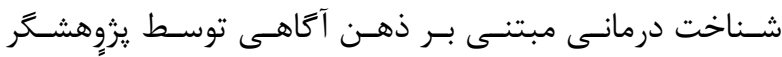

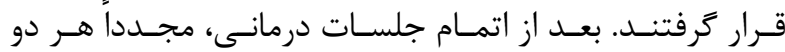

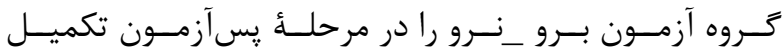

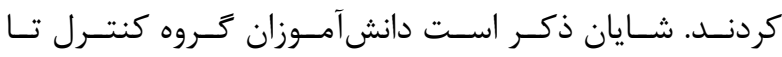

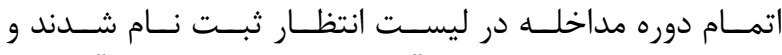

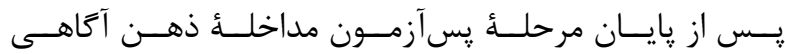

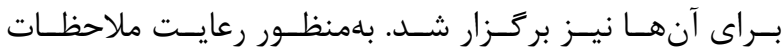

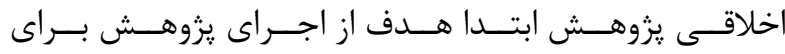

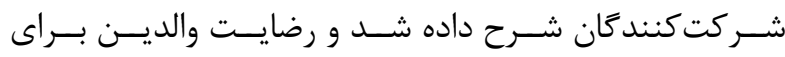

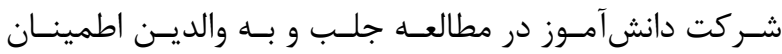

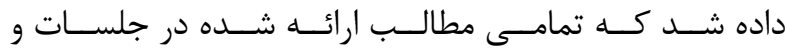

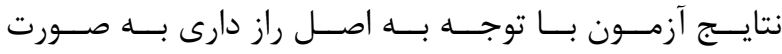

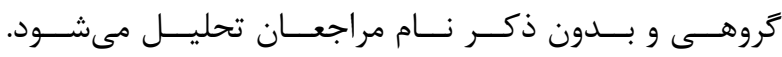

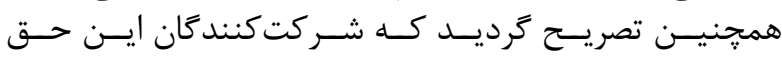

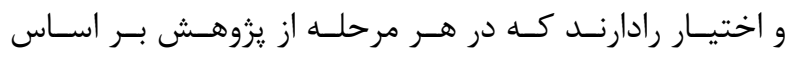

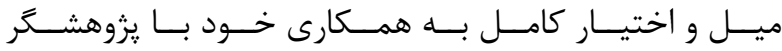

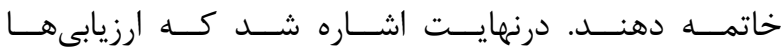

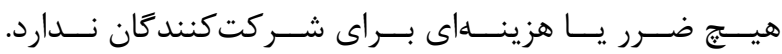

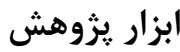

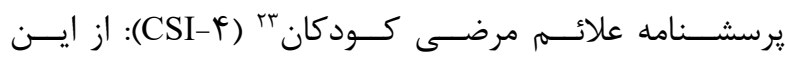

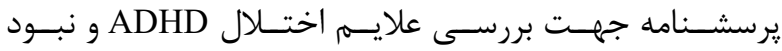

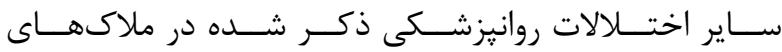

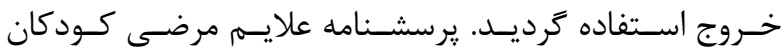

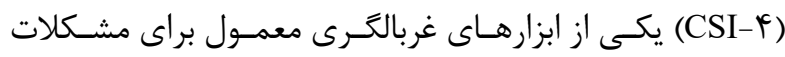

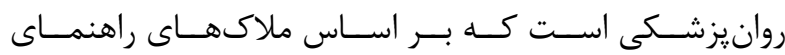

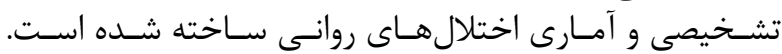

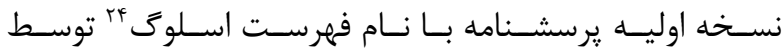

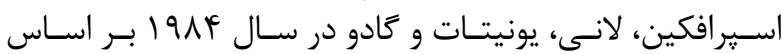

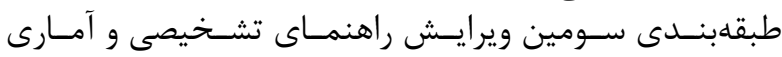

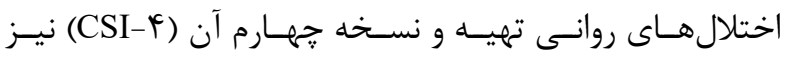

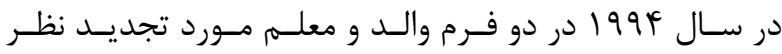

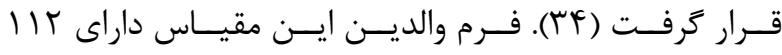

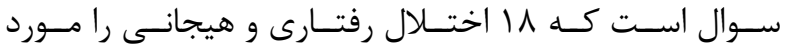

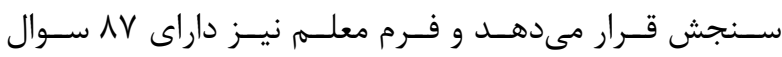

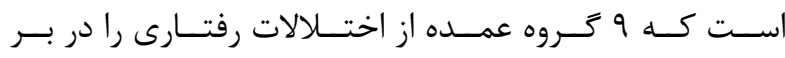

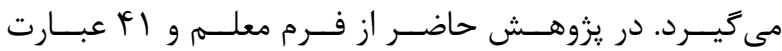

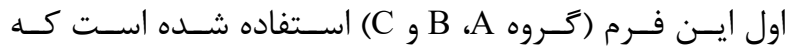

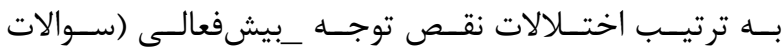

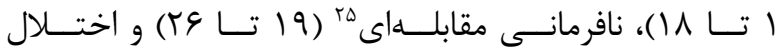

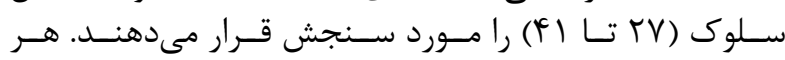

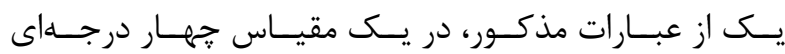

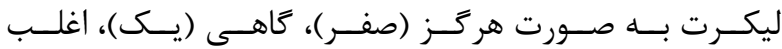

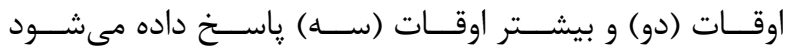

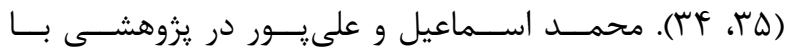


آزمايسش • • •

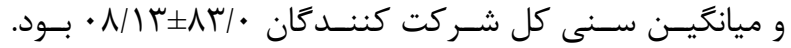

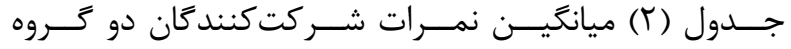

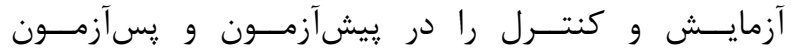

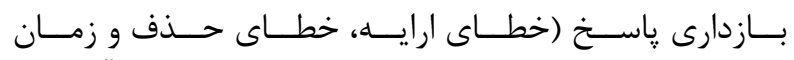

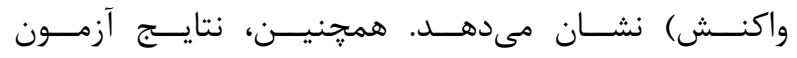

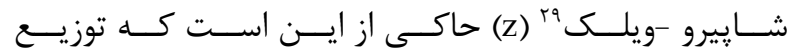

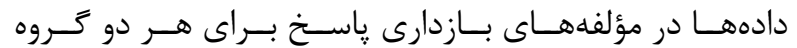

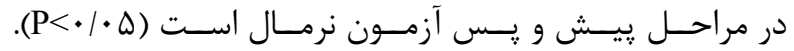

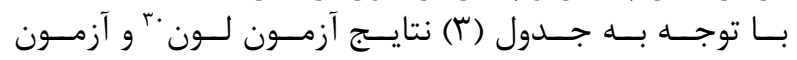

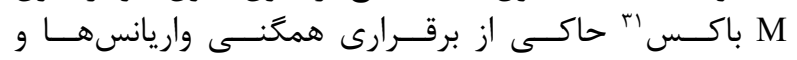

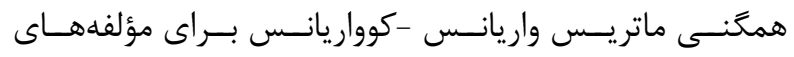

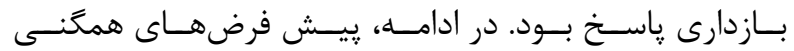

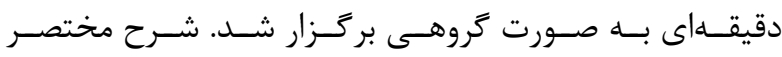

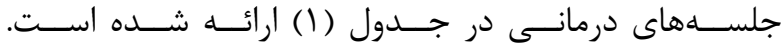

\section{تجزيهوتحليل دادهها}

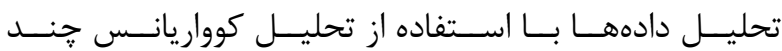

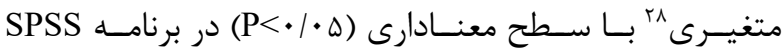

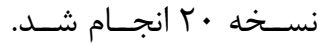

يافته ها

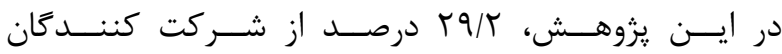

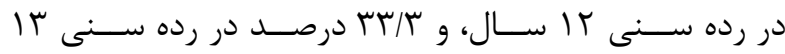

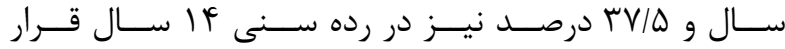

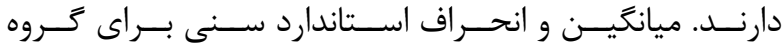

جدول ا- محتواى بسته مداخلة ذهن آَاهى

\begin{tabular}{|c|c|}
\hline محتوا & جلسات \\
\hline برقرارى ارتباط و مفهومسازى، لزوم استفاده از مداخله ذهن آكاهى و آشنايى با هدايت خودكار، أشنايى با هراقبه & اول \\
\hline أموزش مراقبه وارسى بدن، انجام تنغس أكامانه و مراقبه ذهنى، تمركز در مورد تمرينهاو حس كردن دقيق آنها & دوم \\
\hline 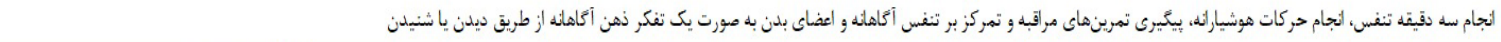 & سوم \\
\hline 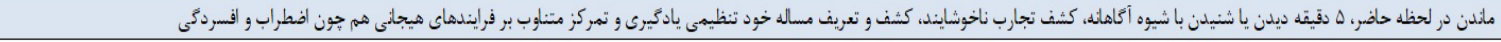 & جهارم \\
\hline 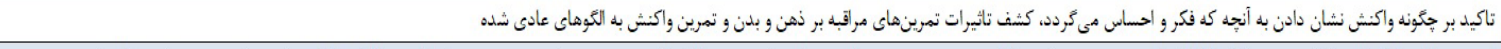 & بنجم \\
\hline 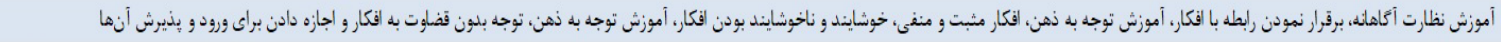 & ششم \\
\hline 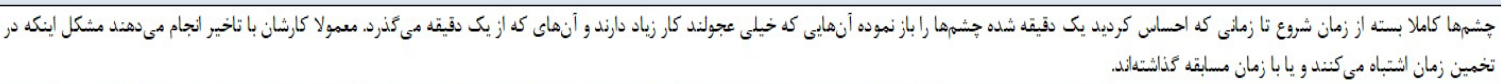 & هicت \\
\hline 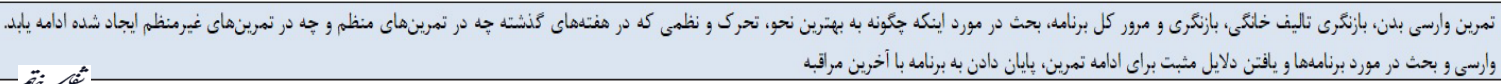 & \\
\hline
\end{tabular}

جدول r- ميانكَين و انحراف استاندارد مؤلفههاى بازدارى ياسخ و نتايج آزمون شإيرو -ويلك در دو كروه آزمايش و كنترل

\begin{tabular}{|c|c|c|c|c|c|c|c|c|}
\hline معنادرى & آمار z & أحراف استاندارد & ميانكين & حداكثر & حداقل & مرحله & كروه & متغير وابسته \\
\hline. $\mid k r$ &.$/ 94$ & $1 / \pi 4$ & $\Delta / 9 r$ & 1 & $\varphi$ & بيش آزمون & \multirow{2}{*}{ آزمايش } & \multirow{4}{*}{ خطاى ارايه } \\
\hline$\cdot \pi \Lambda$ & . $/ 94$ & $I / N \mid$ & RiTd & v & $r$ & يَس آزمون & & \\
\hline.$\pi r$ & .191 & $1 / \cdot 0$ & $\Delta V \Delta$ & 1 & 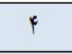 & بيش آزمون & \multirow{2}{*}{ كنترل } & \\
\hline$\cdot|\lambda|$ & .199 & $1 / \pi \Lambda$ & $\Delta / F r$ & 1 & $r$ & بـ آزمون & & \\
\hline$\cdot 1 \cdot 1$ & $\cdot / M$ & $1 / 1 V$ & $V / \Delta$. & 9 & 9 & بيش آزمون & \multirow{2}{*}{ آزمايش } & \multirow{4}{*}{ خطاى حذف } \\
\hline.$\pi 9$ & . $/ 94$ & $1 / \Delta$ & r/ar & 1 & $r$ & يس آزمون & & \\
\hline.$\mu r$ & .94 & I/FF & $V / \cdot 1$ & 9 & $\Delta$ & بيش آزمون & \multirow{2}{*}{ كنترل } & \\
\hline .111 & .19. & $1 / \cdot r$ & $911 \mathrm{~V}$ & 1 & 4 & بس آزمون & & \\
\hline$\cdot / 19$ &.$/ 9 \mathrm{~V}$ & Five & $\mid r V / \Lambda T$ & 190 & ir. & بيش آزمون & \multirow{2}{*}{ آزماش } & \multirow{4}{*}{ زمان واكنش } \\
\hline .1 .9 & $\cdot 1 / 9$ & $N \mid 91$ & $\| K N \cdot \cdot$ & 14. & 119 & بـ آزمون & & \\
\hline .149 & .99 & $r / A Y$ & $|r \Delta| F r$ & IfT & $1 \%$. & بيش آزمون & \multirow{2}{*}{ كنترل } & \\
\hline$\cdot|\lambda|$ & .199 & $4 / .9$ & $1 \pi r / r \Delta$ & 14. & ITS & بـ آزمون & & \\
\hline
\end{tabular}

جدول بـ- نتايج همكنى واريانسها و همًنى ماتريس واريانس -كواريانس براى مولفهاى بازدارى ياسخ

\begin{tabular}{|c|c|c|c|c|c|}
\hline \multirow{2}{*}{ آزمون M باكس براى مولفه هاى بازهارى ياسخ } & \multicolumn{4}{|c|}{ 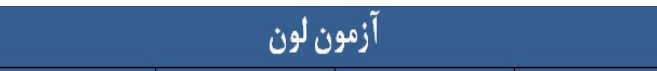 } & \multirow{2}{*}{ متفير وابسته (بازهارى ياسخ) } \\
\hline & معناهارى & df2 & dfi & $\mathrm{F}$ & \\
\hline M= باكس M= 1/11 &.$/ 49$ & rr & 1 &.$/ 19$ & خطاى ارائه \\
\hline$F=1 / f r$ & .144 & rr & 1 & $\cdot \mid \Delta \varphi$ & 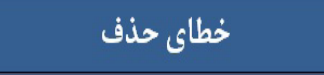 \\
\hline F & $\cdot / 1 \mu$ & rr & 1 & T/FF & زمان واكنش \\
\hline
\end{tabular}

${ }^{28}$ Multivariate analysis of covariance

${ }^{30}$ Leveners test

${ }^{29}$ Shapiro-wilk test

${ }^{31}$ Box>s M test 


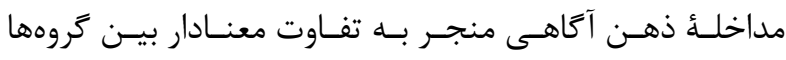

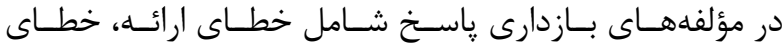

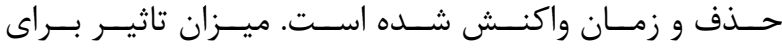

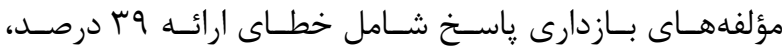

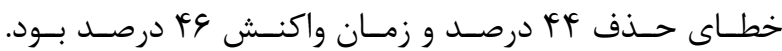

$$
\text { بحث و نتيجهَيرى }
$$

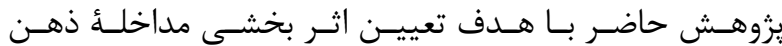

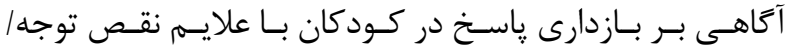

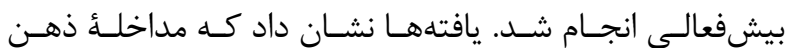

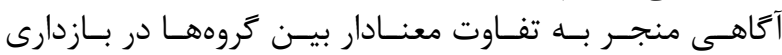

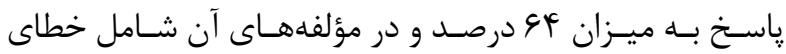

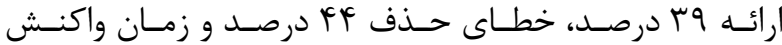

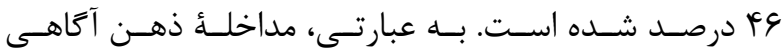

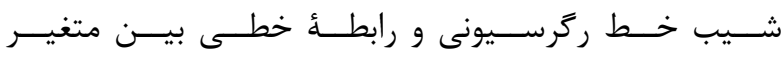

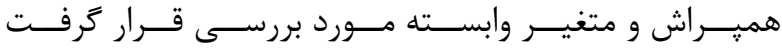

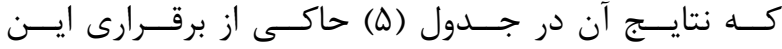

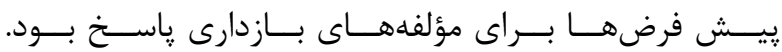

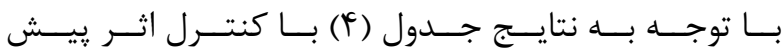

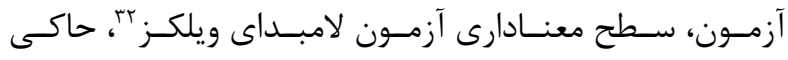

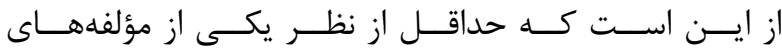

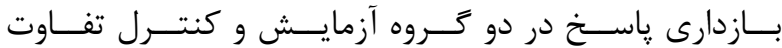

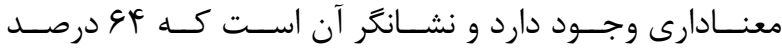

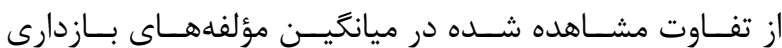

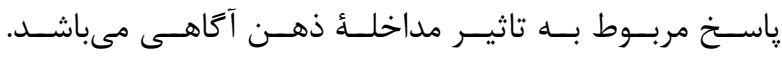

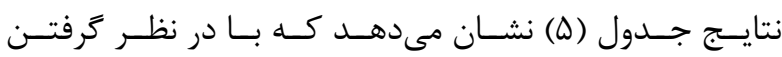

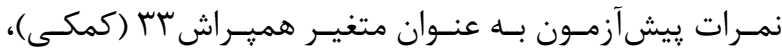

جدول F - نتايج آزمونهاى خند متغيرى براى دو گروه در مولفههاى بازدارى ياسخ

\begin{tabular}{|c|c|c|c|c|}
\hline ضريباتا & معناهارى & $\mathrm{F}$ & الرزش & نام أزمون \\
\hline .194 & $.1 . .1$ & $q / 9 q$ & .194 & لامبداف ويلكز \\
\hline. $\mid 94$ & $.1 . .1$ & $q / q f$ & . $\mu 4$ & اثر يلايى \\
\hline .194 & $.1 . .1$ & $q / 9 q^{c}$ & IND & اترهتلينكا \\
\hline .194 & $.1 . .1$ & $q / q q^{f}$ & IND & بزوكترين ريشه روى \\
\hline
\end{tabular}

جدول ه- نتايج تحليل كواريانس اثرات بين گروهى ميانگَين مولفههاى بازدارى پاسخ در دو گروه

\begin{tabular}{|c|c|c|c|c|c|c|c|}
\hline ضريب اتا & سطع معنادارى & $\mathrm{F}$ & ميانكين مجذورات & درجه آزادى & مجموع مجذورات & منبع تفييرات & متفير \\
\hline$\% r$ & - $/ A<q$ & $\cdot 1 \cdot 4$ & $\cdot 1 \cdot \varphi^{t}$ & 1 & $\cdot 1 \cdot \varphi^{t}$ & كَوهوإيش أزمون & \multirow{4}{*}{ خطاى ارايه } \\
\hline$\cdot / \Delta \varphi$ & $\cdot|\cdots|$ & Tr/VY & $r d / 4$. & 1 & $r d / 4$. & هيش أزمون & \\
\hline \multirow[t]{2}{*}{$\cdot / 49$} & $* \cdots r$ & IT/Y & $1 Y / \Delta D$ & 1 & $1 \% / \Delta \Delta$ & كرو & \\
\hline & & & $1 / \cdot r$ & 19 & $19 / \Delta r$ & خطا & \\
\hline$\cdot / 14$ & $\cdot|| r \mid$ & $r / 91$ & $r / I V$ & 1 & $r / I V$ & كروهيّشيش أزمون & \multirow{4}{*}{ خطاى حنف } \\
\hline 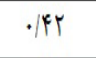 & $\cdot \cdot \cdot \cdot 1$ & $\mid T / A T$ & $14 / 94$ & 1 & $1 \pi / q 4$ & يشي أزمون & \\
\hline \multirow[t]{2}{*}{$\cdot / k p$} & $\cdot 1 \cdots 1$ & $\mid 0 / 1$. & W/Tr & 1 & W/T/T & كرون & \\
\hline & & & $1 / \cdot$ & 19 & $19 / 10$ & خطا & \\
\hline .1 .9 & . 1 rrq & $1 / \Delta G$ & Tr/VA & 1 & rT/VA & كروهميشي أزمون & \multirow{4}{*}{ زمان واكتش } \\
\hline$\cdot 109$ & $\cdot|\cdots|$ & $r V / T$. & $\Delta F \mid / \Lambda 1$ & 1 & $\Delta F \mid / \Lambda 1$ & קيش آزمون & \\
\hline.$/ 14$ & $\cdot \cdot \cdot 1$ & $19 / 49$ & TYATY & 1 & TTDTY & كروه & \\
\hline : & & & $19 / 1 F$ & 19 & $r V V \mid \cdot 1$ & خطا & \\
\hline
\end{tabular}




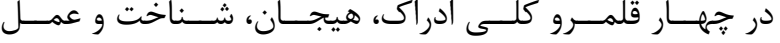

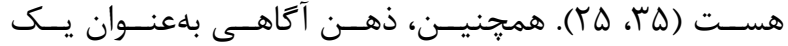

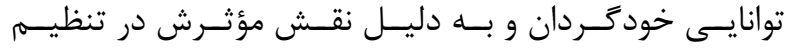

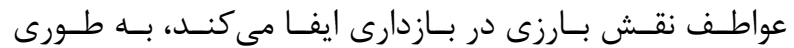

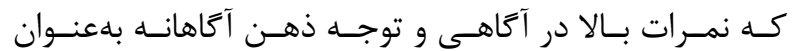

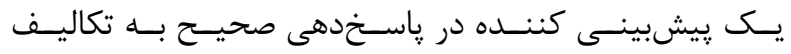

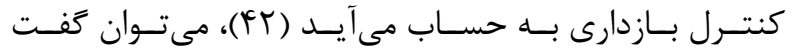

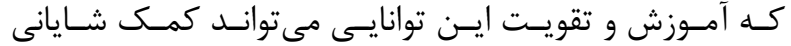

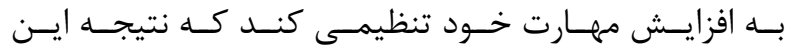

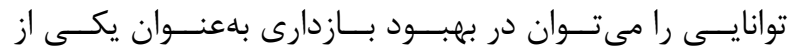

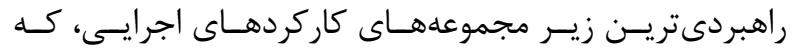

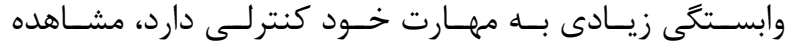

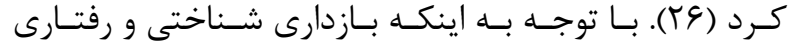

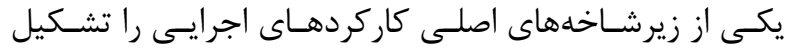

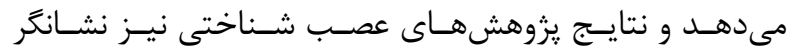

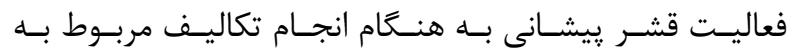

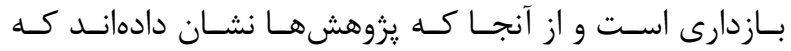

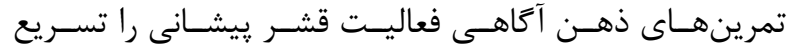

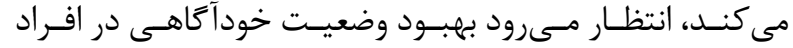

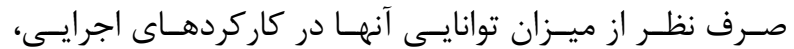

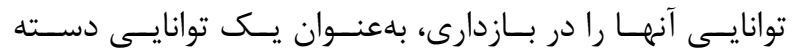

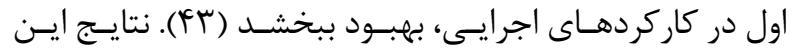

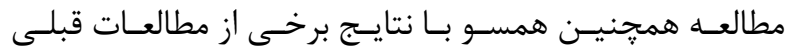

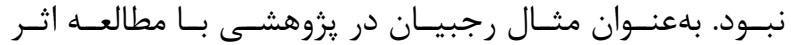

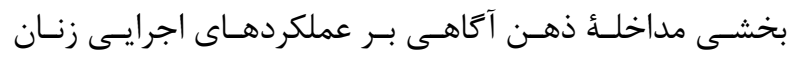

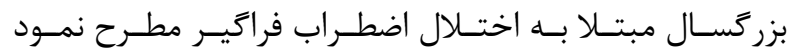

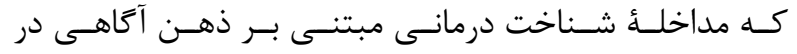

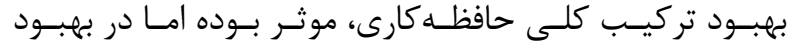

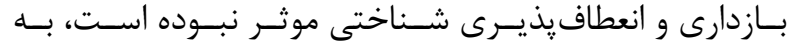

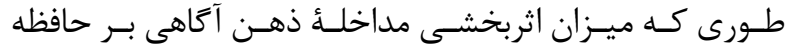

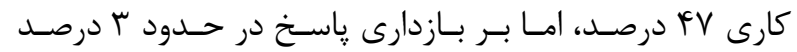

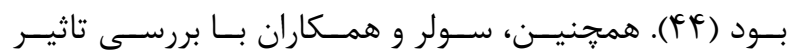

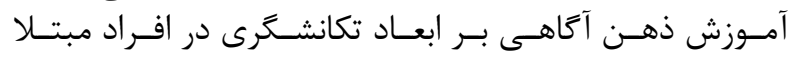

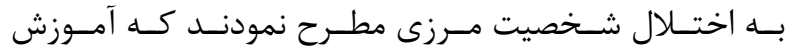

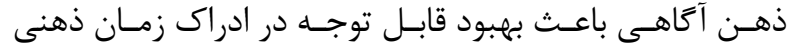

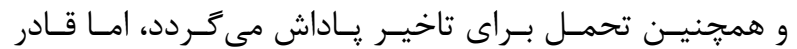

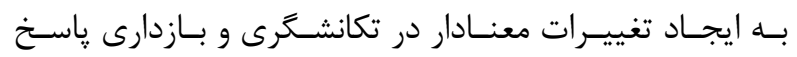

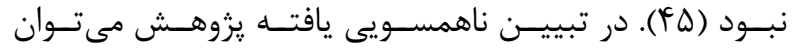

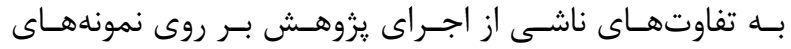

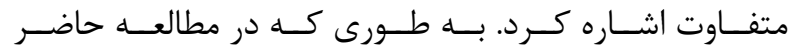

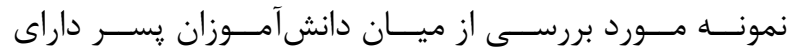

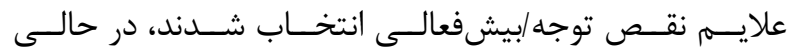

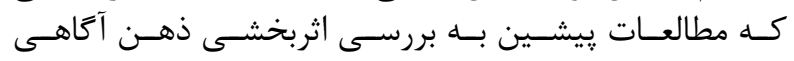

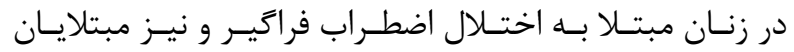

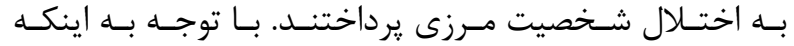

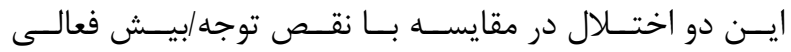

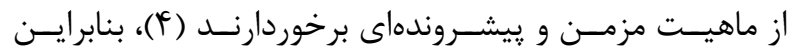

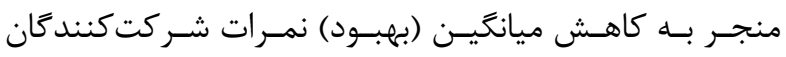

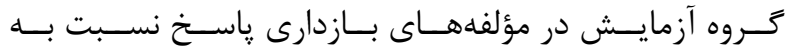

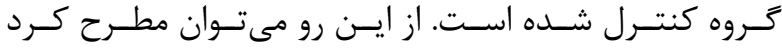

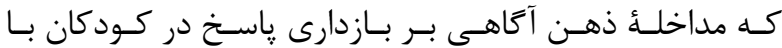

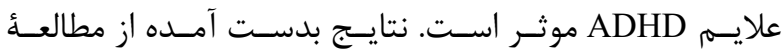

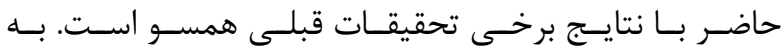

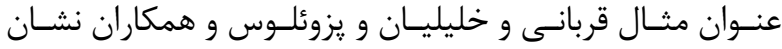

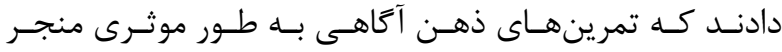

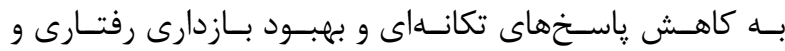

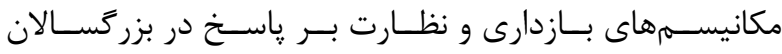

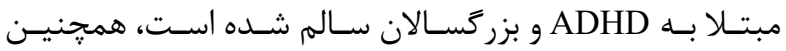

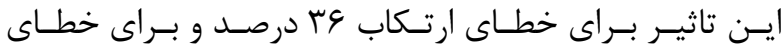

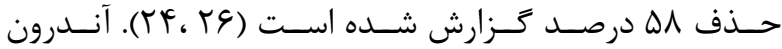

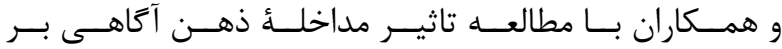

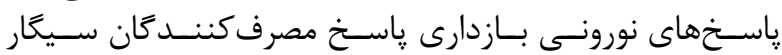

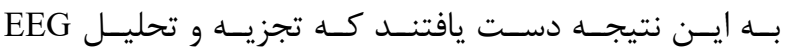

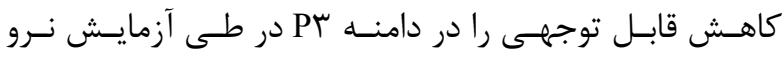

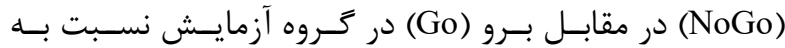

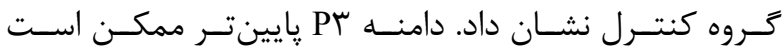

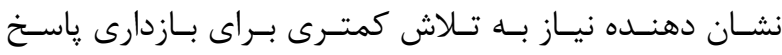

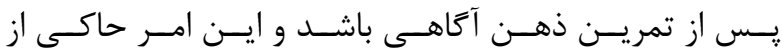

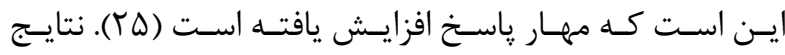

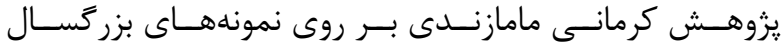

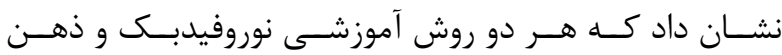

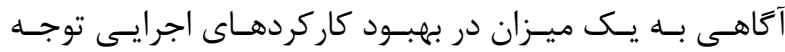

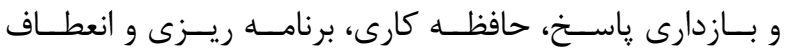

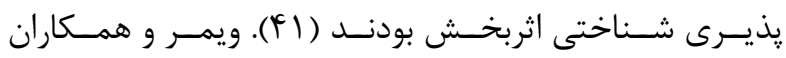

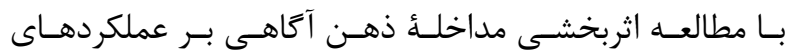

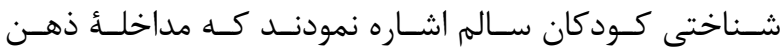

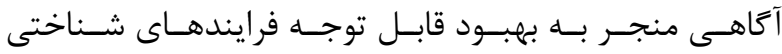

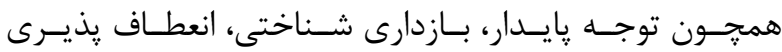

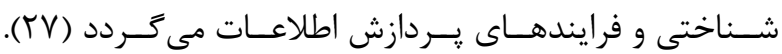

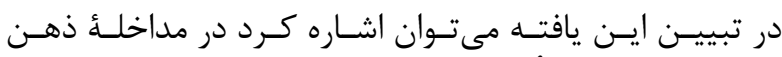

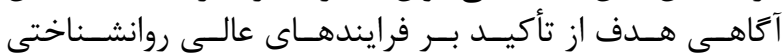

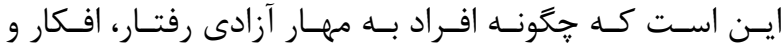

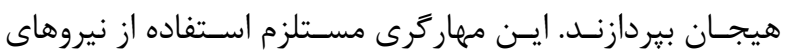

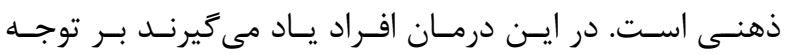

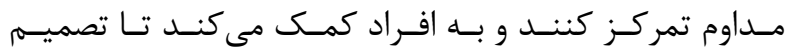

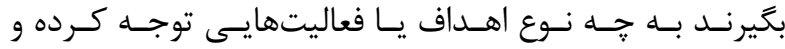

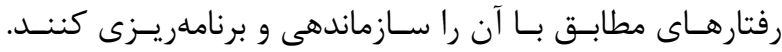

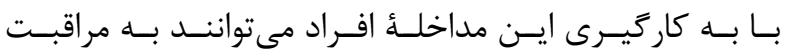

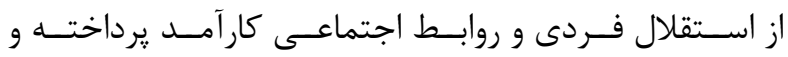

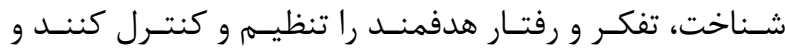

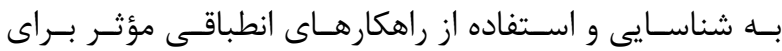

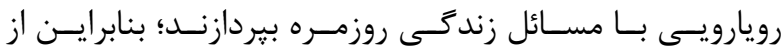

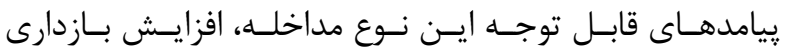




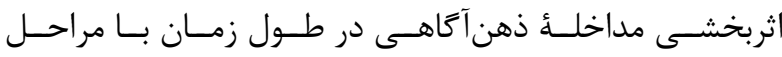

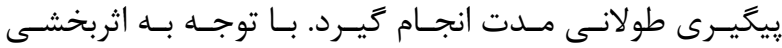

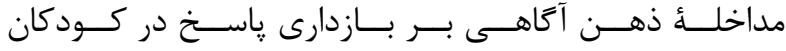

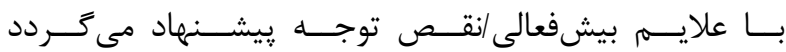

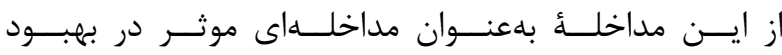

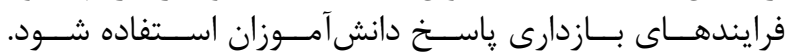

1. Stern L, \& Schell M. Management of AttentionDeficit Disorder and Attention-Deficit/Hyperactivity Disorder Drug Intoxication in Dogs and Cats. Veterinary Clinics of North America: Small Animal Practice. 2018; 48(6): 959-68.

2. Lester GE, Murrell AR. Mindfulness Interventions for College Students with ADHD: A Multiple Single Case Research Design AU - Lester, Ethan G. Journal of College Student Psychotherapy. 2018; 1-22. doi:10.108 0/87568225.2018.1450107

3. Willcut E. The Prevalence of DSM-IV AttentionDeficit/ Hyperactivity Disorder: A Meta- Analytic Review. Neurotherapeutics: the journal of the American Society for Experimental. 2012; 9(3): 490-99.

4. American Psychiatric Association. Diagnos tic and statis tical manual of mental disorders DSM V (5th ed.) Arlington, VA: Author; 2013.

5. Hoseinzadeh Z, Mashhadi A, Ghanaei Cham Abad A, Soltanifar A, Moharerri F, Hoseini Z, \& Hoseinzadeh H. The Combination of Parent Training Program, Working Memory Training on Inhibition in Children with Attention Deficit Hyperactivity Disorder. Research in Clinical Psychology and Counseling. 2014; 3(2): 95 114. [Persian]

6. Barkley RA. Attention Deficit Hyperactivity Disorder: A Handbook for Diagnosis and Treatment. New York Guilford; 2006.

7. Mattison RE, \& Mayes SD. Relationships between learning disability, executive function, and psychopathology in children with ADHD. J Atten Disord. 2012; 16(2): 138-46.

8. Rahbar Karbasdehi E, Hossein Khanzadeh A, Rahbar Karbasdehi F. The Effect of Cognitive-Behavior Anger Management Training on Response Inhibition in Students with Conduct Disorder. Exceptional Education.

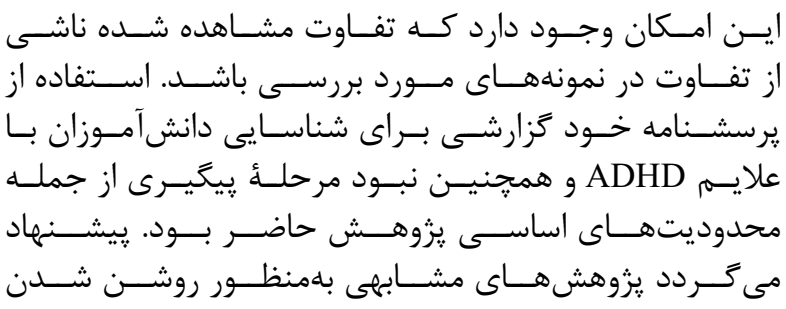

منابع

2017; 3(146): 9-14. [Persian]

9. Wang LC, Tasi HJ, Yang HM. Cognitive inhibition in students with and without dyslexia and dyscalculia. Res Dev Disabil. 2012; 33(5): 1453-461.

10. Robatmili S, Borjali A, Alizadeh H, Nokni M, Farokhi N. Computer- assis ted Cognitive Rehabilitation for response inhibition in children with ADHD (inattentive presentation). Psychology of Exceptional Individuals. 2015; 5(19): 1-126

11. Pooragha Roodbardeh F, Kafi S M, Sotodeh Novroodi $\mathrm{SO}$. Analysis of attention disorder and response inhibition among autism spectrum disorders mental retardation and normal children, J Kermanshah Univ Med Sci. 2013; 17(1): e77144.

12. Bessette KL, Pearlson GD, \& Stevens MC. Effect of trait anxiety on cognitive tes t performance in adolescents with and without attention deficit/hyperactivity disorder AU - Ruf, Barbara M. Journal of Clinical and Experimental Neuropsychology. 2017; 39(5): 434-48. [Persian]

13. Hwang S, Meffert H, Parsley I, Tyler PM, Erway AK, Botkin ML, Blair RJR. Segregating sus tained attention from response inhibition in ADHD: An fMRI s tudy. NeuroImage: Clinical. 2019; 21: 101677.

14. Lee HJ, Kim EH, Yum MS, Ko TS, \& Kim HW. Attention profiles in childhood absence epilepsy compared with attention-deficit/hyperactivity disorder. Brain and Development. 2018; 40(2): 94-99.

15. Griffiths KR, Quintana DS, Hermens DF, Spooner C, Tsang TW, Clarke S, \& Kohn MR. Sus tained attention and heart rate variability in children and adolescents with ADHD. Biological Psychology. 2017; 124: 11-20.

16. Brown KW, Creswell JD, Ryan RM. The evolution of mindfulness research. In: Brown KW, Creswell JD, 
Ryan RM, editors. Handbook of mindfulness: Theory, research, and practice. New York: Guilford; 2015. pp. 13-19.

17. Creswell JD, Lindsay EK. How does mindfulness training affect health? A mindfulness s tress buffering account. Current Directions in Psychological Science. 2014; 23(6): 401-07.

18. Kabat-Zinn J. Wherever you go, there you are:Mindfulness meditation in everyday life. New York, NY: Hachette Books; 2009.

19. Nejati V, Zabihzadeh A, Nikfarjam M. The relationship of mindfulness with sus tained and selective attentional performance. Research in Cognitive and Behavioral Sciences. 2012; 2(2): 31-42. [Persian]

20. Hillgaar SD. Mindfulness And Self Regulated Learning. Mas ter's Thesis in Social and Community Psychology. Trondheim; 2011.

21. Schoenberg PLA, Hepark S, Kan CC, Barendregt HP, Buitelaar JK, \& Speckens AEM. Effects of mindfulnessbased cognitive therapy on neurophysiological correlates of performance monitoring in adult attention-deficit/ hyperactivity disorder. Clinical Neurophysiology. 2014; 125(7): 1407-416.

22. Mitchell JT, Zylowska L, \& Kollins SH. Mindfulness meditation training for attention-deficit/hyperactivity disorder in adulthood: Current empirical support, treatment overview, and future directions. Cognitive and Behavioral Practice. 2015; 22(2): 172-91.

23. Bishop SR, Lau M, Shapiro S, Carlson L, Anderson ND, Carmody J, Segal ZV, Abbey S, Speca M, Velting D, Devins G. Mindfulness: A Proposed Operational Definition. Clinical Psychology: Science and Practice. 2004; 11: 230-41.

24. Pozuelos JP, Mead BR, Rueda MR, \& Malinowski P. Short-term mindful breath awareness training improves inhibitory control and response monitoring. In Progress in Brain Research. 2019; Elsevier.

25. Andreu CI, Cosmelli D, Slagter HA, \& Franken IHA. Effects of a brief mindfulness-meditation intervention on neural measures of response inhibition in cigarette smokers. PLOS ONE. 2018; 13(1), e0191661. doi:10.1371/journal.pone.0191661
26. Ghorbani M, Khalilian R. Effects of Mindfulness Training on Working Memory and Behavioral Inhibition for Adults with Attention Deficit/Hyperactivity. Advances in Cognitive Science. 2016; 18(3): 90-100. [Persian]

27. Wimmer L, Bellingrath S, \& von Stockhausen L. Cognitive Effects of Mindfulness Training: Results of a Pilot Study Based on a Theory Driven Approach. Frontiers in psychology. 2016; 7: 1037. doi:10.3389/fpsyg.2016.01037

28. Nasri S, Nazari MA, Maiqani E, Shahrokhi H, Goodarzi I. Risk-taking and behavioral inhibition interaction in ADHD- with and without conduct disordercompared with control children. Shenakht Journal of Psychology and Psychiatry. 2018; 5(4): 63-77. [Persian]

29. Lee CSC, Ma MT, Ho HY, Tsang KK, Zheng YY, \& Wu ZY. The effectiveness of mindfulness-based intervention in attention on individuals with ADHD: A sys tematic review. Hong Kong Journal of Occupational Therapy. 2017; 30: 33-41.

30. Hazlett-Stevens H. Dispositional Mindfulness and Neural Correlates of Affect Regulation, Arch Neurosci. 2017; 4(3): e57682. doi: 10.5812/archneurosci.57682.

31. Sanger KL, \& Dorjee D. Mindfulness training with adolescents enhances metacognition and the inhibition of irrelevant s timuli: Evidence from event-related brain potentials. Trends Neurosci. 2016; 5(1): 1-11. doi:https:// doi.org/10.1016/j.tine.2016.01.001

32. Bailey NW, Freedman G, Raj K, Sullivan CM, Rogasch NC, Chung SW, Fitzgerald PB. Mindfulness meditators show altered dis tributions of early and late neural activity markers of attention in a response inhibition task. bioRxiv. 2018; 396259. doi:10.1101/396259

33. Pourmohamadi S, Bagheri F. Effectiveness of Mindfulness Training on Automatic Cognitive Processing. Journal of Psychological Studies, 2015; 11(3): 141-159. [Persian]

34. Salami F, Ashayeri H, Estaki M, Farzad V, Koochak Entezar R. Mother-Based Combined Training and Its Effect on the Symptoms Attention Deficit/ Hyperactivity Disorder (ADHD). Neuropsychology. 2016; 2(4): 31-44. [Persian]

35. Najafi M, Foladchang M, Alizadeh H, Mohammadifar 
M. Prevalence of Attention Deficit Hyperactivity Disorder, Conduct Disorder and Oppositional Defiant Disorder. Journal of Exceptional Children. 2009; 9 (3): 239-54. [Persian]

36. Mohamadesmaiel E, Alipour A. A Preliminary Study on the Reliability, Validity and Cut Off Points of the Disorders of Children Symptom Inventory-4 (CSI-4). Journal Of Exceptional Children. 2002; 2(3): 239-54. [Persian]

37. barghi Irani Z, bakhti M, bagiyan agiyankulemare M. The Effectiveness of a Cognitive Processing-based Social Skills Training on the Effectiveness of Cognitive Processing Based Training of Social Skills on, Emotional, Psychological Well-being and Reducing the Symptoms of Children with Conduct Disorder. Social Cognition. 2015; 4(1): 157-75. [Persian]

38. Sohrabi F, Asadzadeh H, Arabzadeh Z. Effectiveness of Barkley's parental education program in reducing the symptoms of oppositional defiant disorder on preschool children. Journal of School Psychology. 2014; 3(2): 82103. [Persian]

39. Verbruggen F, Logan, G. D. Response inhibition in the stop signal paradigm. Trends in cognitive sciences. 2008; 12(11): 418-24. [Persian]

40. Ghadiri F, Jazayeri A, A'shayeri H, Ghazi-Tabatabaei M. The Role of Cognitive Rehabilitation in Reduction of Executive Function Deficits and Obsessive-Compulsive Symptoms in Schizo-Obsessive Patients. Archives of Rehabilitation. 2007; 7(4): 11-24. [Persian]
41. Kermani Mamazandi Z. Comparing the effectiveness of neurofeedback training and mindfulness training on improving executive functions (attention and response retention, working memory, cognitive planning and flexibility) in athletic students. Doctoral dissertation, Semnan University; 2018. [Persian]

42. Manikavasgar V, Parker G, Perich T. Mindfulness based cognitive therapy \& cognitive bahavioral therapy as a treatment for non-menalcholic depression. Journal of Affective Disorders. 2010; 30: 138-44.

43. Ryan MA, Priddy SE, Riquino MR, Froeliger B, Howard MO. Effects of mindfulness-oriented recovery enhancement versus social support on negative affective interference during inhibitory control among opioidtreated chronic pain patients: a pilot mechanis tic study, Annals of Behavioral Medicine. 2019; 53(10): 865-76.

44. Azam Rajabian A. The effectiveness of mindfulnessbased cognitive therapy in improving working memory, inhibition, and cognitive flexibility in people with generalized anxiety disorder. MSc Thesis, Ferdowsi University of Mashhad; 2017. [Persian]

45. Soler J, Elices M, Pascual JC, Martín-Blanco A, Feliu-Soler A, Carmona C, \& Portella MJ. Effects of mindfulness training on different components of impulsivity in borderline personality disorder: results from a pilot randomized study. Borderline Personality Disorder and Emotion Dysregulation. 2016; 3(1): 1-10. 